\title{
Systematic Index
}

Section 2: Mesodermaptera sectio nova $\ldots \ldots \ldots \ldots \ldots \ldots \ldots \ldots, 1$

Superfamily Carcinophoroidea Sternmann $\ldots \ldots \ldots \ldots \ldots \ldots \ldots, 1$

2. Family Carcinophoridae PoPHAm..................... 2

1. Subfamily Platylabiinae BURR $\ldots \ldots \ldots \ldots \ldots \ldots \ldots \ldots \ldots, 6$

Genus 1: Platylabia DoHRN ................................ 6

1. P. major DoHRN ............................... 7

2. Subfamily Gonolabiinae Popham \& Brindle................ 8

Genus 2: Gonolabina VerHOEfF ........................ 8

1. G. spectabilis (PHILIPPI) ............................. 9

2. G. trinodosa BRINDLE ............................. 10

3. G. binodosa BRINDLE ............................. 11

3a. G. binodosa bidonosa BRINDLE .......................... 11

3b. G. binodosa llanguena BRINDLE......................... 12

3. Subfamily Anophthalmolabiinae Steinmann. .............. 13

Genus 3: Anophthalmolabis BrindLE $\ldots \ldots \ldots \ldots \ldots \ldots \ldots \ldots \ldots \ldots$

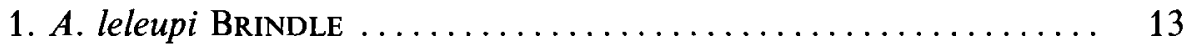

2. A. caeca (BoRELLI) ............................. 14

4. Subfamily Carcinophorinae Hincks ................... 15

Genus 4: Epilandex Hebard............................. 17

1. E. solomonensis BRINDLE......................... 19

2. E. handschini HINCKs. .......................... 20

3. E. peterseni RamamurTHI ........................ 21

4. E. undulata RAMAMURTHI ........................ 22

5. E. burri (BoRELLI) .............................. 23

6. E. bazyluki SteInMANn ........................ 23

Genus 5: Titanolabis BurR ............................ 25

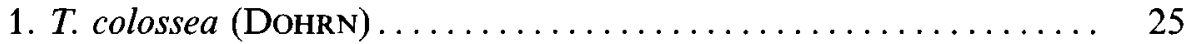

2. T. gigas sp.n............................... 26 
VIII

Genus 6: Capralabis Brindle.......................... 27

1. C. ashmolei BRINDLE ............................ 28

2. C. steinmanni Brindle............................... 29

3. C. srivastavai Brindle............................ 29

Genus 7: Zacheria Steinmann ........................ 30

1. Z. polita (ZACHER) .............................. 30

2. Z. dentata (BURR) ........................... 31

Genus 8: Thekalabis KAPOOR .......................... 32

1. T. genitalis KAPOOR ............................ 32

Genus 9: Indolabis gen. n........................... 33

1. I. papua sp. n. ............................... 34

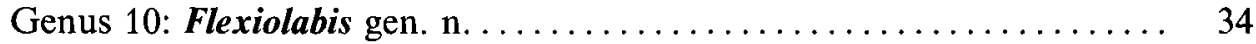

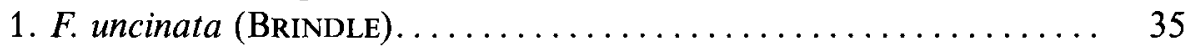

2. F. hamata (BRINDLE) ......................... 36

Genus 11: Paraflexiolabis gen. $n . \ldots \ldots \ldots \ldots \ldots \ldots \ldots \ldots \ldots \ldots, 37$

1. P. ornata sp. n. .............................. 37

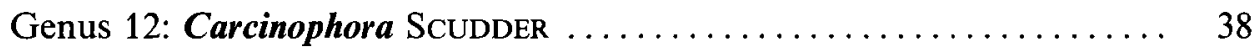

1. C. spitzi (Menozzi) ................................ 40

2. C. boesemani Steinmann ........................ 41

3. C. festae (Borelli) ........................... 42

4. C. peruviana (Bormans) ......................... 43

5. C. apolinari (HEBARD) ............................ 44

6. C. festiva (BURR)............................... 45

7. C. waddyi Burr................................. 45

8. C. venezuelica BrINDLE ......................... 46

9. C. burri (BoRELLI) ........................... 47

10. C. percheroni (Guérin \& Percheron)................ 48

11. C. americana (Palisot de Beauvois) ................... 49

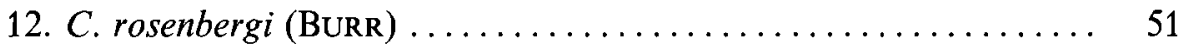

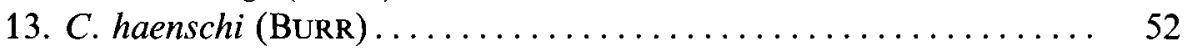

14. C. minima (MoreIRA)............................ 53

15. C. scudderi (Bormans) ............................ 53

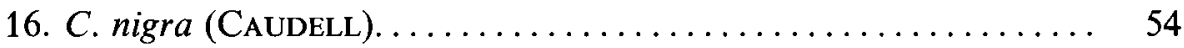

17. C. gagatina (KLUG) .............................. 55

18. C. brasiliensis (MoREIRA) ........................ 56

19. C. occidentalis (KIRBY) ............................. 57

20. C. venusta $\mathrm{sp}$. nov............................. 58

21. C. kawakamii (SHIRAKI) ........................... 59

22. C. castetsi Bormans........................... 59 
Genus 13: Anisolabis FIERER........................... 60

1. A. maritima (BoNELLI) .......................... 69

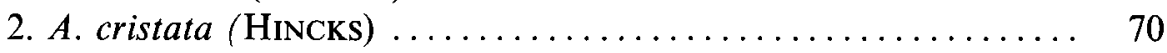

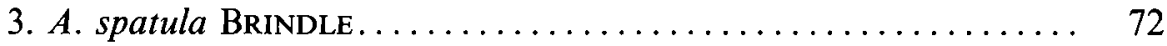

4. A. cunicula Brindle............................ 73

5. A. porrectella Brindle.......................... 74

6. A. rostrata Brindle .............................. 75

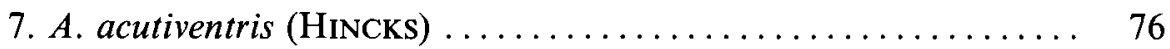

8. A. oweni BURR............................... 77

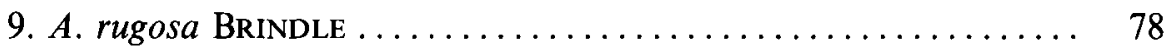

10. A. pluto ReHN ................................ 80

11. A. incisa BoreLLI .............................. 80

12. A. incisiodes BrindLE............................. 82

13. A. quadricollis (HINCKs) ............................ 83

14. A. felix Burr............................... 84

14a. A. felix felix BURR .............................. 85

14b. A. felix elgonensis BrindLE........................ 86

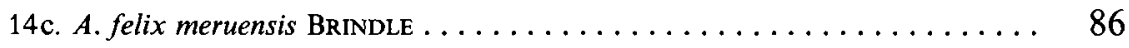

14d. A. felix paradoxura (ZACHER) $\ldots \ldots \ldots \ldots \ldots \ldots \ldots \ldots \ldots \ldots \ldots \ldots \ldots$

14e. A. felix chyuluensis BrindLE ......................... 86

15. A. bintumanensis BRINDLE....................... 87

16. A. hottentotta (DoHRN) ........................ 88

17. A. rufescens KIRBY .................................. 89

18. A. atra Borelli................................. 90

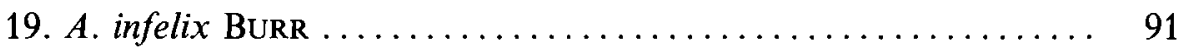

20. A. straeleni (Hincks) ............................ 92

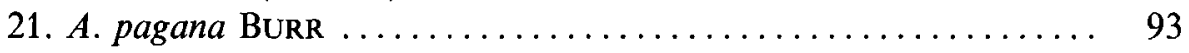

22. A. duplicata nom. nov. ...................... 94

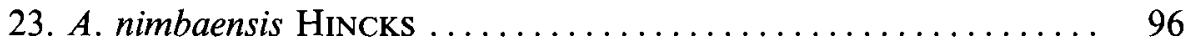

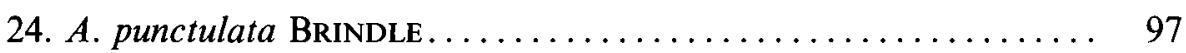

25. A. jeanneli (MenozzI) ............................ 98

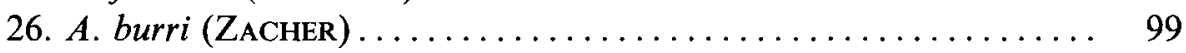

27. A. scotti BrindLE ................................ 100

28. A. laeta (Gerstaecker) ......................... 101

29. A. tumida Borelli ............................ 102

30. A. royi BrindLE............................... 103

31. A. turgida BURR .............................. 104

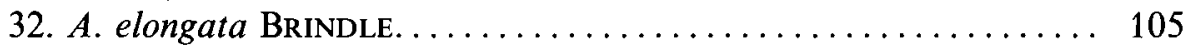

33. A. isomorpha BoRELLI ......................... 106

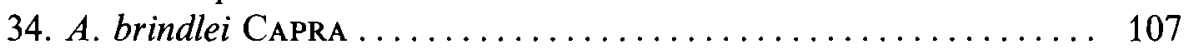

35. A. neavei BRINDLE. ............................ 108

36. A. gestri BoRELli.......................... 109 
37. A. vosseleri BURR ............................ 110

38. A. caesarea (ZACHER)........................... 111

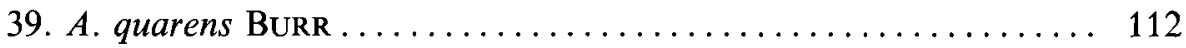

40. A. lamottei Brinde............................. 113

41. A. rougemonti BRINDLE .......................... 114

42. A. kristenseni BURR ............................. 115

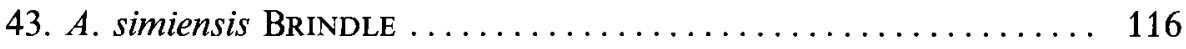

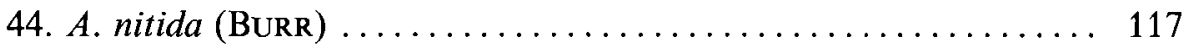

45. A. australis Tindale............................ 117

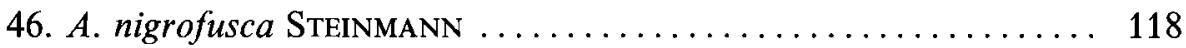

47. A. flavocapitata Steinmann ....................... 119

48. A. littorea (White).............................. 120

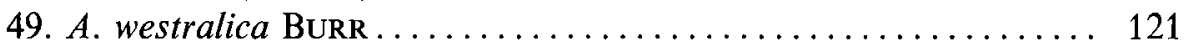

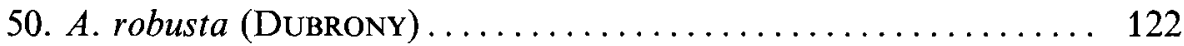

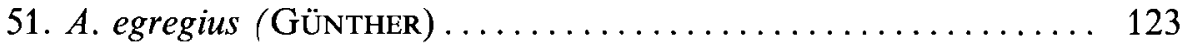

52. A. perissa GüNTHER ........................... 124

53. A. horvathi BURR ............................. 125

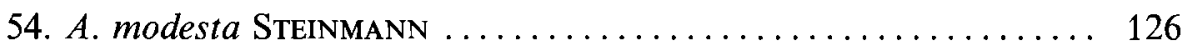

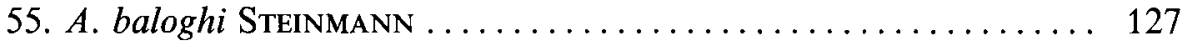

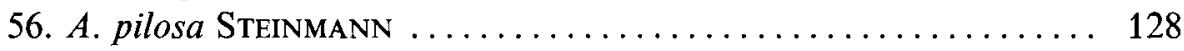

57. A. bifida BrindLE ................................ 129

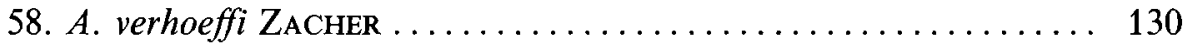

59. A. canaca BrindLE ............................... 131

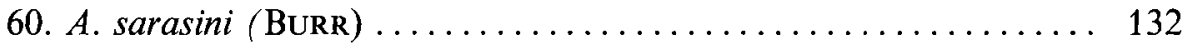

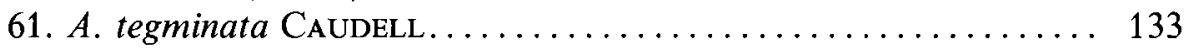

62. A. minutissima Brinde......................... 133

63. A. pacifica (ERICHSON) ............................ 134

64. A. subarmata (KIRBY) ........................... 136

65. A. pectoralis (EsCHSCHOLTZ) ....................... 136

66. A. ryukyuensis (NISHIKAWA) ...................... 137

67. A. montshadskii Bey-BiEnKo ........................ 138

68. A. aborensis (BURR) ........................... 139

69. A. antennata (RAmAMURTHI \& DAVID)................. 140

70. A. lefroyi (BURR) ............................. 141

71. A. dohrni (KIRBY) ............................. 142

72. A. orientalis (RAMAMURTHI) ....................... 144

73. $A$. mira (BEY-BIENKo) ........................... 145

74. A. dubronyi KIRBY .............................. 145

75. A. vitalisi BURR .............................. 146

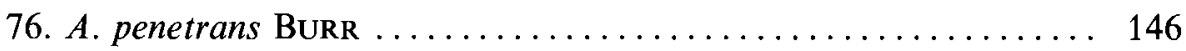

77. A. greeni BURR .............................. 147

78. A. recurva BoRELLI............................ 148 
79. A. gaudens BUrR.............................. 148

80. A. maindrioni (BoRELLI).......................... 149

81. A. rubella BRINDLE .............................. 150

82. A. kudagae BurR ............................ 151

Genus 14: Foramenolabis Steinmann. .................... 152

1. F. sisera (BURR) .............................. 153

2. F. afghana SteinMANN ......................... 154

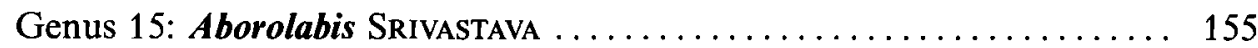

1. A. nepalensis (BRINDLE)......................... 156

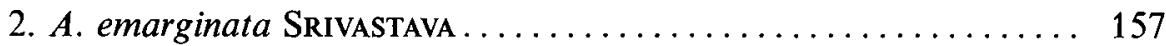

3. A. kalaktangensis SRIVASTAVA ...................... 158

4. A. pervicina (BURR) ............................. 159

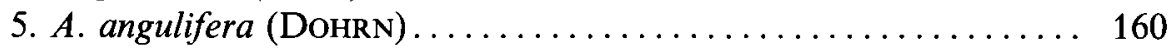

6. A. vicina (BURR) .............................. 161

7. A. cerrobarjai Steinmann .......................... 162

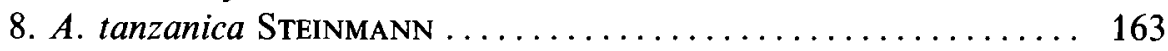

9. A. mordax Steinmann ............................. 164

10. A. mauritanica (LuCAS) ........................... 165

Genus 16: Ornatolabis gen. nov......................... 166

1. O. externa (BEY-BIENKo) ........................ 167

Genus 17: Anisolabella ZACHER ............................. 168

1. A. braueri ZACHER .............................. 168

Genus 18: Heterolabis BORELLI . . . . . . . . . . . . . . . . . . . . . . 169

1. H. brasiliensis BORELLI............................. 169

Genus 19: Epilabis Burr................................ 170

1. E. nilgiriensis SRIVASTAVA......................... 172

2. E. harlequin nom. nov. .......................... 172

3. E. penicillata (BoRELLI) ........................... 173

4. E. emarginata RamamurThi \& DAVID ................... 174

5. E. analis Ramamurthi \& DAVID ......................... 175

6. E. punctata SRIVAstava ............................ 177

7. E. burri SRIVASTAVA ................................ 178

8. E. ramachandrai RAMAMURTHI \& DAVID.................. 179

9. E. vallakadaiensis RAMAMURTHI \& DAVID................ 179

Genus 20: Metalabis BURR ............................. 180

1. M. ecarinata BRINDLE ........................... 181

2. M. brasiliensis BRINDLE........................... 182

3. M. saramaccensis (ZACHER) ....................... 183 
4. $M$. deplanata ReHn............................. 184

5. $M$. carinata BRINDLE .......................... 185

Genus 21: Gonolabis Burr ............................ 186

1. G. electa BURR................................. 190

2. G. splendida Steinmann ........................... 191

3. G. deserta SteInMANn ......................... 192

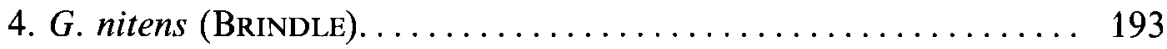

5. G. puella StEINMANN ............................ 194

6. G. silvestrii (BORELLI)............................. 195

7. G. nigeriensis (BRINDLE) ........................... 196

8. G. sinuata (BRINDLE) ............................ 197

9. G. laevigata (BRINDLE)............................. 197

10. G. transversalis (BRINDLE).......................... 198

11. G. hincksi (BRINDLE)............................ 199

12. G. tanganyikae (BRINDLE) ........................ 200

13. G. umbrosa (BRINDLE) ........................... 201

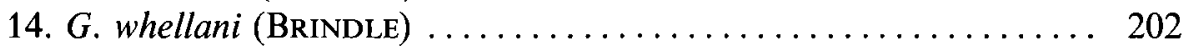

15. G. maxima (BRULLE) .......................... 203

16. G. marginalis (DoHRN) ........................... 204

17. G. distincta (NISHIKAWA) ........................ 205

18. G. bidens SteinMann ........................... 207

19. G. gilesi StEINMANn .............................. 207

20. G. dentata Steinmann .......................... 208

21. G. javana (Bormans) ............................. 209

22. G. forcipata BURR .............................. 210

23. G. tasmanica (BoRMAns) ........................... 211

24. G. blanchardi (GUILLON) ......................... 212

25. G. woodwardi BURR ............................. 213

26. G. sechuana (BEY-BIENKo).......................... 214

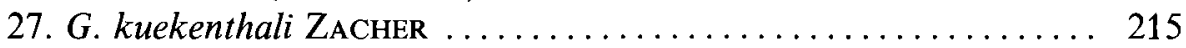

28. G. cavaleriei (BORELLI) ............................ 216

29. G. panayica Steinmann .......................... 217

30. G. fallax (BEy-BIENKo) ......................... 218

31. G. formosae (BoRELLI) .......................... 219

32. G. mystica SteinMANn ......................... 220

33. G. hwangi (BEY-BIENKo) ........................ 221

34. G. insidiata Steinmann .......................... 222

35. G. insulana BRINDLE............................. 223

36. G. acuta BOESEMAN .............................. 224

37. G. undata (BEY-BIENKo) .......................... 225

38. G. kirbyi (BURR) ............................ 227 
39. G. darevskyi BeY-Brenko...................... 227

40. G. magna (BEY-BIENKo)........................... 228

41. G. panfilovi (BEY-BIENKO) .......................... 229

? G. minor BoRELLI ............................... 230

Genus 22: Euborellia BuRr.............................. 231

1. E. vanderbilti REHN ............................ 237

2. E. cincticollis (GERSTAECKER) ..................... 238

3. E. moesta (GÉNÉ) ........................... 239

4. E. flavohumeralis BRINDLE ...................... 240

5. E. ituriensis BRINDLE ......................... 241

6. E. stali (DoHRN) ............................. 242

7. E. purpurea (BoRELLI) ......................... 244

8. E. fulviceps BoRELLI ............................ 244

9. E. feae (BoRELLI) . . . . . . . . . . . . . . . . . . . . . . . 245

10. E. andreinii (BoRELLI) .......................... 246

11. E. dimidiata BRINDLE .......................... 247

12. E. flava SteInMAnN............................. 248

13. E. compressa (BoRELLI) ........................... 249

14. E. ugandana BrindLE............................ 250

15. E. annulipes (LuCAs) .......................... 251

16. E. tellinii (BoRELLI).............................. 253

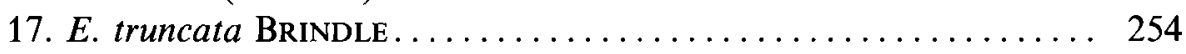

18. E. peregrina (MJÖBERG) ......................... 254

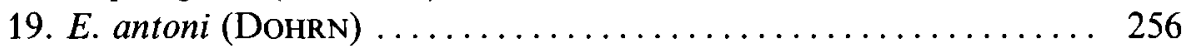

20. E. uruguayensis BRINDLE........................ 257

21. E. mexicana BRINdLE............................ 258

22. E. armata (BORELLI) .......................... 259

23. E. plebeja (DoHRN) ............................. 259

24. E. maxima MoReIRA ........................... 261

25. E. nitida MoreIRA............................. 261

26. E. flavipes MoreIRA ............................ 262

27. E. boliviana BRINDLE ........................... 263

28. E. ambigua (BoRELLI)............................ 264

29. E. caraibea HeBARd .............................. 264

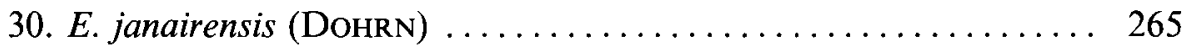

31. E. tatei Hebard............................... 266

32. E. femoralis (DoHRN) ........................... 267

33. E. insulana (BORELLI) ........................... 269

34. E. malgacha BRINDLE.............................. 269

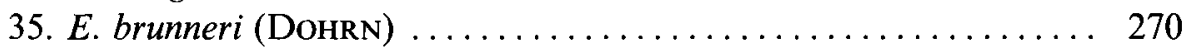

36. E. eteronoma (BORELLI) ........................ 271 
37. E. aporonoma (BORELLI) ....................... 272

38. E. dattai Srivastava ........................... 273

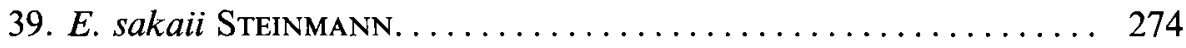

40. E. rajasthanensis Srivastava ...................... 275

41. E. punctata BoRELLI........................... 276

42. E. abbreviata SRIVASTAVA............................ 277

43. E. annandalei (BURR) ......................... 278

44. E. philippinensis SrivaStava ...................... 279

5. Subfamily Brachylabiinae BURR $\ldots \ldots \ldots \ldots \ldots \ldots \ldots \ldots \ldots \ldots 280$

Genus 23: Metisolabis Burr............................ 281

1. M. submetallica (BRINDLE) ....................... 282

2. M. milloti (HINCKs) ............................ 283

3. $M$. voeltzkowi (BURR).......................... 284

4. $M$. malgacha (BURR) ........................... 285

5. $M$. bifoveolata (Bolivar)......................... 286

6. $M$. punctata (DUBRONY) .......................... 287

7. M. fulgens sp. nov.......................... 288

Genus 24: Ctenisolabis VerhoefF ........................ 289

1. C. togoensis VerHOEFF ............................. 291

2. C. ruficollis (Hincks) .......................... 292

3. C. traegaordhi (BURR) ........................... 293

4. C. montana (BORELLI) ........................... 294

5. C. nigra (SCUDDER)............................. 294

6. C. mahunkai SteInMANN .......................... 295

7. C. pusilla Steinmann ............................. 296

8. C. aciculata Steinmann.............................. 297

9. C. fletcheri BuRR .............................. 298

10. C. loebli Steinmann ............................. 299

Genus 25: Brachylabis DoHRn............................... 299

1. B. lenkoi BRINDLE ............................ 301

2. B. chilensis (BLANCHARD) ....................... 301

3. B. coriacea BuRR............................. 302

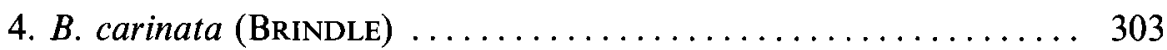

5. B. fuscula BRINDLE .............................. 304

6. B. yaloma RAMAMURTHI ......................... 305

7. B. canaca BURR ................................. 306

8. B. manawatawhi GiLEs........................ 307

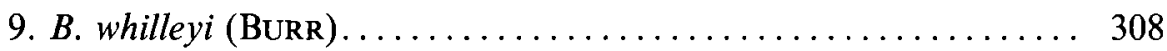

10. B. tegminata SteInMANn .......................... 309

11. B. philetas BURR ........................... 309 
?. B. collinsi BrindLE .............................. 310

6. Subfamily Isolabiinae Steinmann $\ldots \ldots \ldots \ldots \ldots \ldots \ldots \ldots \ldots \ldots, 311$

Genus 26: Pterolabis gen. nov. ....................... 312

1. P. villiersi (BRINDLE) .......................... 312

Genus 27: Isolabis VeRHOEFF ......................... 313

1. I. proxima (BRINDLE) ........................... 315

2. I. transverse BRINDLE ......................... 316

3. I. unicolor (BRINDLE) . . . . . . . . . . . . . . . . . . . . . . . 317

4. I. bicolor (BRINDLE) ........................... 319

5. I. grandicollis (HINCKs) ........................ 320

6. I. sjoestedti (BORG) ............................. 321

7. I. attenuata BRINDLE ........................... 322

8. I. coiffaiti (BRINDLE)................................ 323

9. I. schoutedeni HINCKS ........................... 324

10. I. usumbarana (VERHOEFF) .............................. 325

11. I. rufa (BRINDLE) .................................. 326

12. I. quadrata BRINDLE ............................ 327

13. I. braueri VeRHOEFF........................... 328

14. I. pauliani (HiNCKs) . ............................ 329

15. I. howardi (BURR) ........................... 330

16. I. ocellata SRIVASTAVA......................... 331

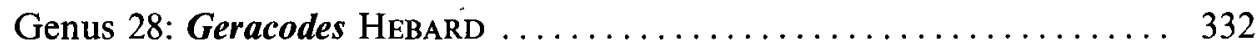

1. G. litus HeBard ............................... 333

2. G. paraguayensis BoRELLI ........................ 334

3. G. labioides HincKs ............................ 334

4. G. aptera BRINDLE.......................... 335

Genus 29: Africolabis Brindle ...................... 336

1. A. pseudohumicola BrindLE ...................... 337

2. A. humicola (BRINDLE).......................... 338

7. Subfamily Antisolabiinae BRINDLE .................... 339

Genus 30: Antisolabis BURR ............................. 339

1. A sulcatipes BURR........................... 342

2. A. meridionalis (BRINDLE) ....................... 343

3. A fusca (BRINDLE)............................ 344

4. A. myrmecoides BurR .......................... 345

5. A. camerunensis (BoRELLI) ...................... 346

6. A. rugosa (BRINDLE) ............................ 347

7. A. minima (BRINDLE) ............................. 348

8. A. scotti (BURR) ............................ 349 


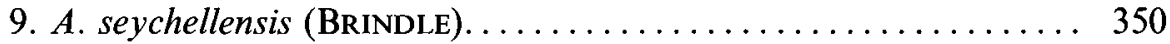

10. A. notabilis Sternmann ........................ 351

11. A. holdhausi (BURR) .......................... 352

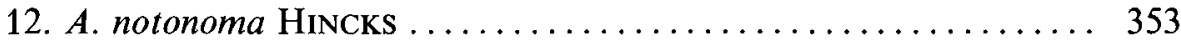

13. A. gisleni (Hıncks) ............................ 354

14. A. solitaria sp. n............................... 355

15. A. venusta sp.n............................. 356

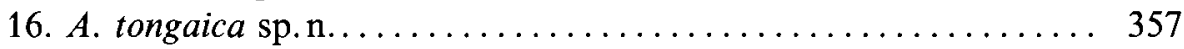

17. A. fijica sp.n.............................. 358

18. A. cordata (BRINDLE) ........................... 358

19. A. greensladei (BRINDLE) ........................... 359

20. A. rouxi Burr .............................. 360

21. A. rouxiodes (BRINDLE) ....................... 361

22. A. arripiens BURR .......................... 362

23. A. forficula (BURR) ........................... 363

24. A. geniculata (MONTROUZIER) ..................... 364

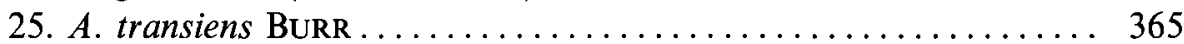

26. A. allardi (BRindLE) ............................ 366

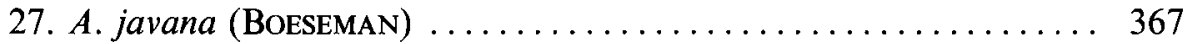

28. A. ceylonica (BRINDLE) ......................... 368

29. A. formicoides (BURR) . . . . . . . . . . . . . . . . . . 369

30. A. dammermani (BORELLI). ..................... 369

31. A. kelangi (BRINDLE) .......................... 370

8. Subfamily Parisolabiinae Verhoeff $\ldots \ldots \ldots \ldots \ldots \ldots \ldots \ldots \ldots . \ldots \ldots$

Genus 31: Parisolabis VerHoEfF .......................... 372

1. P. renschi (GüNTHER) ........................... 373

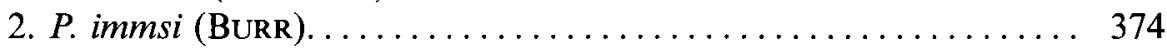

3. P. tenera (BURR) .............................. 375

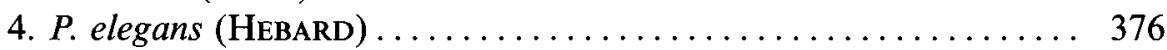

5. P. setosa Hudson ................................ 376

6. P. johnsi Hudson ............................... 377

7. P. iti Hudson ................................... 378

8. P. tapanuiensis Hudson............................ 379

9. P. forsteri Hudson ............................ 380

10. P. novaezeelandiae VERHOEFF ...................... 381

11. P. nelsonensis Hudson ... . . . . . . . . . . . . . . . . . . . . . . . . 382

12. P. boulderensis Hudson ........................ 383

Genus 32: Parisopsalis BUrR ........................... 384

1. P. spryi BURR ................................ 384

9. Subfamily Idolopsalinae Steinmann $\ldots \ldots \ldots \ldots \ldots \ldots \ldots \ldots \ldots 386$ 


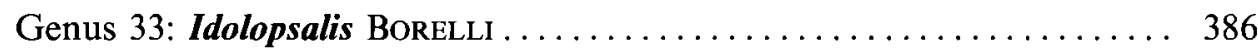

1. I. borgmeieri Menozzı............................ 387

2. I. azteca (DoHRN) ............................... 388

3. I. andeana BURR ............................... 389

4. I. koepckei BRINDLE ................................ 390

5. I. gracilis BRINDLE ............................. 391

6. I. whymperi BURR ............................... 391

7. I. incerta (BoRmANs)............................. 392

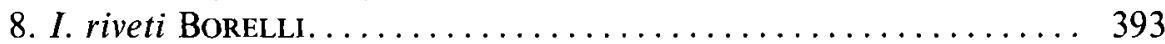

9. I. nigrita BRINDLE .............................. 394

10. I. curta BRINDLE ................................. 395

11. I. parva Brindle.............................. 396

3. Family Labiduridae VerhoEFF .......................... 398

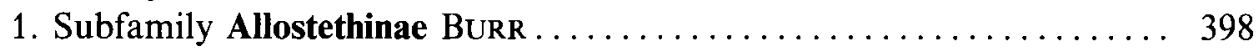

Genus 1: Gonolabidura ZACHER ............................... 399

1. G. javana BoESEMAN .......................... 400

2. G. boschmai BoESEMAN. ......................... 401

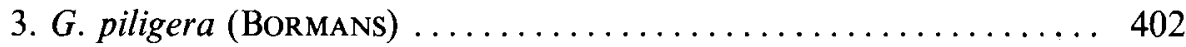

4. G. astruci BuRR ................................. 403

5. G. nathani BRINDLE........................... 404

6. G. minor BURR .................................. 405

Genus 2: Allostethus VERHOEFF ......................... 406

1. A. minus (Bormans) ............................. 407

2. A. philippinense Borelli ........................ 408

3. A. burri Brindle ........................... 409

4. A. indicum (BURMEISTER) .......................... 409

5. A. setiger VeRHOEFF ........................... 411

6. A. gracilie BRINDLE........................... 412

7. A. celebense BURr.............................. 413

8. A. lombokianum VeRHOEFF ........................ 414

9. A. anamalayanum Ramamurthi .................... 415

Genus 3: Allostethella ZACHER........................... 416

1. A. nitens ZACHER............................. 417

2. A. guttata (Bormans) ........................... 417

3. A. doriae (Dubrony) . . . . . . . . . . . . . . . . . . 418

4. A. sumatrana BrindLE............................ 419

5. A. malayana ZACHER .......................... 420

2. Subfamily Nalinae Sternmann $\ldots \ldots \ldots \ldots \ldots \ldots \ldots \ldots \ldots \ldots . \ldots 21$

Genus 4: Nala ZACHER ................................ 422

1. $N$. lividipes (Dufour)........................ 423 


\section{XVIII}

2. N. caprea MenOzZi .............................. 424

3. $N$. figinii (BURR) ................................. 425

4. $N$. intermedia Menozzi ........................ 426

5. N. saegeri BRINDLE............................ 427

6. $N$. ornata BoRELLI............................. 429

7. $N$. nepalensis (BURR) ............................ 429

8. $N$. timorensis BRINDLE .......................... 431

9. $N$. tenuicornis (Bormans) ........................ 432

10. N. basalis BEY-BIENKo ......................... 433

3. Subfamily Labidurinae BURR ....................... 434

Genus 5: Tomopygia BURR ............................. 435

1. T. abnormis (BORMANs) ......................... 435

Genus 6: Labidura LEACH.......................... 436

1. L. xanthopus (STÅL) ............................... 437

2. L. minor Boeseman............................ 438

3. L. dharchulensis GANGOLA ....................... 440

4. L. herculeana (FABRICIUS) .......................... 441

5. L. japonica (DE HAAN) ........................... 442

6. L. riparia (PALLAS) .............................. 443

7. L. orientalis Steinmann.......................... 446

Genus 7: Forcipula Bolivar ........................ 447

1. F. quelchi BURR ............................. 450

1a. F. quelchi quelchi BURR .......................... 451

1b. F. quelchi boliviana BRINDLE......................... 451

2. F. americana BoRMANS...................... 452

3. F. congo BURR ............................... 453

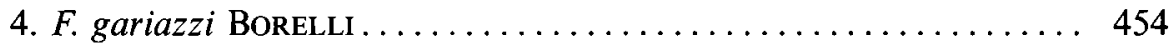

5. F. tanganyikae Hincks. ......................... 455

6. F. tarsata (WeSTWOOD) ............................ 456

7. F. borellii CHOPARD .................................. 457

8. F. lurida Bolivar. ............................... 458

9. F. banksi BoReLli .............................. 459

10. F. turberculata SRIVASTAVA ..................... 460

11. F. leonardii StEINMANn ......................... 461

12. F. quadrispinosa (DoHRN) ....................... 462

13. F. simplex BEY-BIENko........................... 463

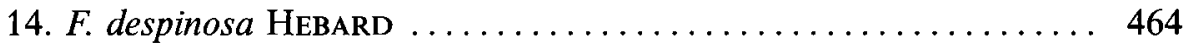

15. F. yunnanea BEY-BIENKo ......................... 465

16. F. trispinosa (DoHRN) ......................... 466

17. F. afghana SternmanN ........................ 467 


\section{XIX}

18. $F$. decolyi Bormans. ......................... 468

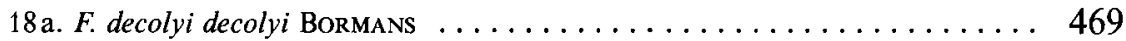

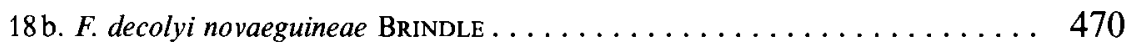

19. F. aborensis BRINDLE ......................... 470

20. F. clavata Liu............................. 471

21. F. walkeri (KIRBY)........................... 473

22. $F$. vanheurni (BOESEMAN) .......................... 474

23. F. indica BRINDLE ........................... 475

24. F. obscura Steinmann ....................... 476

Section 3: Paradermaptera Verhoeff .................. 478

Superfamily Apachyoidea SteinMann.................... 478

4. Family Apachyidae VERHOEFF......................... 478

1. Subfamily Apachyinae BURR ........................ 479

Genus 1: Dendroiketes BUrR .......................... 479

1. D. novaeguineae Boeseman ....................... 480

2. D. corticinus (BURR) ......................... 481

3. D. punctatus BoRELli........................... 482

Genus 2: Apachyus Audinet-Serville ................... 483

1. A. depressus (Palisot de Beauvois) ................. 484

2. A. reichardi KARSCH. .......................... 485

3. A. murrayi DoHRN ............................. 486

4. A. baloghi Steinmann ........................... 488

5. A. feae Bormans ............................. 488

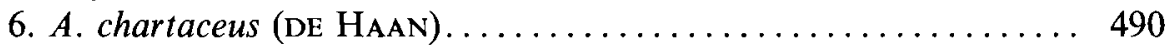

7. A. javanus VerhoEFF................................ 491

8. A. peterseni BoRelli........................... 492

9. A. queenslandicus MJöBERG ..................... 493

10. A. athertonensis MıöвERG........................ 493

11. A. beccari Dubrony.......................... 494

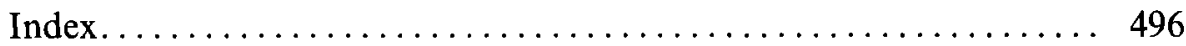


